\title{
A INFLUÊNCIA DO SUPORTE SOCIAL NA VINCULAÇÃO PAI/BEBÉ
}

\author{
Carla Cruz \\ Paula Nelas \\ Emília Coutinho \\ Cláudia Chaves \\ Odete Amaral
}

Escola Superior de Saúde de Viseu - Instituto Politécnico de Viseu - CI\&DETS

cruzcarla@hotmail.com

https://doi.org/10.17060/ijodaep.2017.n1.v3.1018

Fecha de Recepción: 28 Enero 2017

Fecha de Admisión: 1 Abril 2017

\section{RESUMO}

Enquadramento: 0 suporte social é um fator relevante no estabelecimento de laços afetivos entre pai e bebé, pois envolve a qualidade das relações sociais centradas na família. Este tem sido apontado como uma variável psicossocial envolvida na adaptação à parentalidade.

Objetivo: Estabelecer a relação entre o suporte social e a vinculação (bonding) entre o pai e 0 bebé.

Método: Trata-se de um estudo transversal, descritivo-correlacional, de natureza quantitativa, com uma amostra de 349 pais média de idade 31,84 anos, $d p=6,067)$. 0 instrumento de colheita de dados foi o questionário que permitiu caracterizar a amostra nas variáveis sociodemográficas, de gravidez e parto e foi ainda incluída a Escala de Satisfação com o Suporte Social (Ribeiro, 1999) e Escala de Bonding (Figueiredo et al., 2005).

Resultados: Os resultados demonstram que 0 bonding positivo é influenciado pela idade $(p=0,010)$, habilitações literárias $(p=0,045)$, ser primeiro filho $(p=0,027)$, comunicação com 0 feto $(p=0,005)$, presença no trabalho de parto $(p=0,045)$. Quanto ao suporte social foi encontrada relação entre 0 bonding positivo e "satisfação com os amigos" ( $p=0,011)$; bonding negativo e "intimidades" ( $p=0,011)$ e bonding total e "satisfação com a família" ( $p=0,001)$.

Conclusão: 0 papel do enfermeiro especialista em saúde materna assume-se como primordial, devendo envolver o pai desde o início da gravidez. Dada a importância que o suporte social assume no estabelecimento do bonding, é imprescindível que os profissionais de saúde apoiem e integrem 0 pai no processo de adaptação à parentalidade.

Palavras-chave: Pai; Bebé; Trabalho de Parto; Bonding; Suport. 


\section{A INFLUÊNCIA DO SUPORTE SOCIAL NA VINCULAÇÃO PAI/BEBÉ}

\section{ABSTRACT}

The influence of social support on parent/baby bonding

Background: The social support is an important factor in the establishment of bonding between parent and baby, because it involve the quality of social relations in the family. This factor has been pointed out a psychosocial variable involved in adaptation to parenthood.

Objective: Investigate the influence between social support and bonding of father and baby.

Methods: This study is a cross-sectional study, correlational descriptive, quantitative, attended by 349 parents, aged between 19 and 55 , with an average age of 31,84 ( $\mathrm{sd}=6,067$ ). To providing a reliable sample characterization the social-demographic, obstetric and pregnancy variables, we applied questionnaires and also the Satisfaction Scale with the Social Support (Ribeiro, 1999) and Bonding Scale (Figueiredo et al., 2005).

Results: This study reveals that positive bonding is influenced by age $(p=0,010)$, qualifications $(p=0,045)$, first child $(p=0,027)$, communication with fetus $(p=0,005)$ and be present during labor $(p=0,045)$.

Regarding to social support: - Positive bonding and "satisfaction with friends" $(p=0,011)$; Negative bonding and "intimacies" ( $p=0,011)$; - Total bonding and "family satisfaction" $(p=0,001)$.

Conclusion: The data undoubtedly show the importance of nurse specialists in maternal health who must involve the father since the beginning of pregnancy. Since social support is key to bonding, it is essential that health professionals support and integrate the father in the adjustement process to parenthood.

Keywords: Father; Baby; Labor; Bonding; Social Support.

\section{INTRODUÇÃO}

0 nascimento de um bebé, particularmente do primeiro filho, institui-se como um grande impacto no seio familiar. Como tal, o processo de transição para a parentalidade com as respetivas alterações de papéis familiares e 0 aumento das responsabilidades implicam frequentemente momentos de tensão familiar, havendo uma sequência previsível de transformações na organização familiar (RELVAS, 2004).

Estas reorganizações internas, constituem-se sempre como momentos de uma certa ansiedade, stresse quer para a mãe, quer para o pai, gerando por vezes crises (RELVAS, 2004). Da alteração no papel do homem no processo de transição para a parentalidade, origina-se a necessidade de adaptações intra e interpessoais para as quais o pai necessita de orientação e de apoio social, com repercussões no estabelecimento de vínculos afetivos em relação ao recém-nascido.

0 processo de vinculação é indispensável pois irá favorecer e/ou determinar a futura relação entre a díade: pai/bebé, bem como influenciar o desenvolvimento cognitivo e social da própria criança. 0 processo de vinculação (bonding), é condicionado por um conjunto de fatores interligados com as condições parentais e as situacionais que poderão coadjuvar ou impedir o estabelecimento do processo de vinculação (PEREIRA, 2009).

Neste contexto surge 0 conceito de bonding, que remete para a ligação da figura materna e paterna ao recém-nascido. Esta vinculação, no caso do presente trabalho, refere-se a todo $0 \mathrm{com}$ portamento que permite ao pai estabelecer e manter a proximidade ou contacto com o bebé, tratando-se de um fenómeno vital para este, como a respiração ou a satisfação de necessidades humanas básicas (BRANDÃ0, 2009).

A interação estabelecida entre o pai e o bebé promove o bonding, enquanto processo gradativo de envolvimento afetivo entre esta díade, que decorre durante o período de gravidez, nos primeiros momentos após o nascimento do bebé e no pós-parto. Figueiredo et al (2005) salientam que o bon- 
ding não ocorre imediatamente após o nascimento, ou seja, é um processo que se declara e fortalece progressivamente no decorrer do primeiro ano de vida. Para estes autores, a formação do vínculo entre pai e filho costuma ser mais lenta, consolidando-se gradualmente após o nascimento e no decorrer do desenvolvimento da criança.

A este respeito, Carvalho (2003), refere que o pai ao assistir ao nascimento do filho vive experiências positivas pelo suporte emocional que proporciona à companheira/mulher e pelos sentimentos e emoções que experiência, os quais podem favorecer o maior envolvimento emocional precoce com o filho.

0 bonding poderá ser influenciado por aspetos psicossociais, nomeadamente 0 suporte social que o pai dispõe ou perceciona no trabalho de parto e parto. 0 suporte social percebido é uma variável da personalidade que se mantém estável ao longo do tempo, reportando-se à experiência de vinculação. As pessoas que estabeleceram uma vinculação segura terão a perceção de um alto suporte social, crendo que as pessoas que constituem a sua rede social, próxima e alargada, estarão mais disponíveis. A perceção de um suporte social disponível, mesmo não existindo como tal, aumenta o bem-estar do indivíduo, anulando efeitos potencialmente negativos decorrentes do facto da não existência de suporte quando necessário (FREITAS, 2009).

A consciência desta realidade, consubstanciada com a motivação pessoal, aleada às evidências de poucos estudos que se enfoquem no estabelecimento de laços afetivos entre o pai e 0 bebé no trabalho de parto e parto, levou-nos à seguinte questão, subjacente à problemática do presente estudo:

- Será que o suporte social influencia o bonding?

As evidências mostram que o pai quer ser cada vez mais um elemento ativo no momento do parto, considerando fulcral a sua presença enquanto companheiro dando apoio físico e emocional à mãe. Este experiencia sentimentos positivos e negativos que nem sempre vão de encontro ao idealizado, podendo sofrer influência do suporte social de que dispõe.

Estabelecer a relação entre 0 suporte social e a vinculação (bonding) entre 0 pai e 0 bebé será 0 objetivo primordial deste estudo, cujos resultados, esperamos que contribuam para o conhecimento mais profundo sobre o fenómeno do bonding e suporte social e para 0 desenvolvimento de intervenções mais ativas e específicas dos enfermeiros.

\section{MATERIAL E MÉTODOS}

Realizámos um estudo quantitativo, do tipo transversal, descritivo-correlacional, com uma amostra intencional não probabilística por conveniência, constituída por 349 progenitores do sexo masculino, com idade compreendida entre os 19 aos 55 anos, ( $x=31,84$ anos) e ( $d p=6,067)$. A maioria tem com habilitações literárias até ao 3ํㅜ Ciclo $(37,4 \%)$, são casados $(66,9 \%)$ e mantêm uma relação conjugal entre os 4 e os 7 anos (35,8\%).

0 bonding (processo de envolvimento afetivo entre o pai e o bebé) é a variável dependente e 0 Suporte Social (recursos psicológicos e materiais que 0 individuo possui para satisfazer as suas necessidades sociais), a variável independente.

0 instrumento de colheita de dados é composto por um questionário de caracterização sociodemográfica, obstétrica e envolvimento do pai na gravidez, trabalho de parto e parto; Escala de Satisfação com o Suporte Social (ESSS) de Pais Ribeiro (1999), que irá medir a perceção de suporte social, e a Escala de Bonding de Figueiredo et al em 2005, que irá permitir avaliar o envolvimento emocional dos pais com 0 bebé. Esta escala está dividida em três subescalas que avaliam 0 Bonding positivo, Bonding negativo e o Bonding not clear. 0 Bonding Total, que é obtido através da subtração das subescalas "bonding negativo" e "bonding Not Clear" à subescala "bonding Positivo". 


\section{A INFLUÊNCIA DO SUPORTE SOCIAL NA VINCULAÇÃO PAI/BEBÉ}

No decurso do processo de colheita de dados foi garantida a confidencialidade e a privacidade, assim como obtido o consentimento informado do respondente para a participação no estudo, pelo preenchimento livre e esclarecido do questionário.

\section{RESULTADOS}

Os resultados demonstram que 0 bonding positivo é influenciado pela idade $(p=0,010)$, habilitações literárias $(p=0,045)$, ser primeiro filho $(p=0,027)$, comunicação com 0 feto $(p=0,005)$, presença no trabalho de parto $(p=0,045)$. Quanto ao suporte social foi encontrada relação entre 0 bonding positivo e "satisfação com os amigos" ( $p=0,011)$; bonding negativo e "intimidades" $(p=0,011)$ e bonding total e "satisfação com a família" $(p=0,001)$.

\section{DISCUSSÃO}

A adaptação à parentalidade depende do contexto em que 0 individuo se insere, nomeadamente, nas relações que estabelece e nos vários papéis que desempenha refere-nos Moura-Ramos e Canavarro (2007).

Procuramos nesta pesquisa estudar a relação entre 0 suporte social e 0 bonding.

Relativamente ao bonding positivo apuramos que existem correlações positivas e estatisticamente significativas em todas as subescalas do suporte social com o bonding positivo: "satisfação com os amigos" ( $p=0,000)$; "intimidades" ( $p=0,004)$, "satisfação com a família" ( $p=0.017)$ e "actividades sociais" $(p=0,006)$. Deste modo, concluímos que à medida que aumenta satisfação das referidas subescalas, o bonding positivo também aumenta. Aplicando o modelo de regressão múltipla, constatámos que a primeira e única variável a entrar no modelo é a "satisfação com os amigos", explicando por si só $18,1 \%$ do bonding positivo. Aplicando os testes $\mathrm{F}$ e t, verificamos que os resultados são estatisticamente significativos $(p=0,011)$ e pelos coeficientes padronizados inferimos que a satisfação com os amigos é preditiva do bonding positivo.

Deste modo, deduz-se que quanto maior a satisfação com os amigos, maior é o bonding positivo.

Para comprovar a dimensão preditora da "satisfação com os amigos" com o bonding positivo, fundamentamo-nos na ideia de Menino e Sousa (2010) que confirmam ser necessários apoios efetivos, especialmente da família e dos amigos, para promover a relação entre o pai e 0 filho. Referem ainda que este suporte é essencial para que o processo de paternidade se desenvolva harmoniosa e equilibradamente. 0 estudo de Deave et al (2008) também veio confirmar que os progenitores do sexo masculino, durante o período pré e pós-natal, referem sentir maior apoio por parte dos profissionais de saúde e colegas de trabalho.

Os resultados que apuramos permitem-nos concluir a existência de uma correlação positiva entre as subescalas do suporte social e o bonding positivo alicerçados na opinião de Oliveira (2007) que refere que as relações com companheiros, familiares, amigos e colegas de trabalho são importantes para o bem-estar de cada indivíduo. Feijó (2006) Cit. por Oliveira (2007) também afirma que para além da família, existem várias pessoas que participam na rede social e que podem exercer influências importantes em vários momentos da vida. Dessen e Braz (2000) confirmam esta ideia, mencionando que além da família, também a escola, o local de trabalho e os vizinhos interferem nas interações familiares. Desta forma, infere-se que o progenitor masculino pode ter apoio de diversas fontes que tal como os autores supracitados referem influenciam nos momentos de transições familiares, nomeadamente o nascimento de um filho.

Em relação ao bonding negativo, verificámos a existência de correlações negativas em todas as subescalas do suporte social, exceto na "satisfação com os amigos", onde a correlação foi positiva. Analisamos ainda que existem correlações significativas na variável "intimidades" $(p=0,006)$ e 
"satisfação com a família" ( $\mathrm{p}=0,011$ ), ou seja, à medida que aumentam os índices do suporte social, 0 bonding negativo diminui. Aplicando o modelo de regressão multiplica, apuramos que a primeira e única variável a entrar no modelo de regressão são as "intimidades", explicando 13,5 \% do bonding negativo. Aplicando os testes $\mathrm{F}$ e t, constatamos que existem resultados estatisticamente significativos $(p=0,011)$ e pelos coeficientes padronizados inferimos que as "intimidades" têm valor preditivo para o bonding negativo. Logo, deduzimos que quanto maior é a satisfação com as intimidades, menor é o bonding negativo.

Estes resultados vão de encontro ao espectável e são validados por Genesosi et al (2009), afirmando que o contexto social em que os pais vivem e trabalham, bem como a qualidade do relacionamento com a companheira influenciam a transição dos homens para a parentalidade. Parke (1996) Cit. por Boli (2002) também confirma a importância das intimidades, confirmando que 0 relacionamento do casal interfere no envolvimento paterno. Finalmente, Wicki (1999) Cit. por Boli (2002) reconhece que 0 apoio emocional da companheira tem, possivelmente, o maior impacto no bem-estar do pai, durante o processo de adaptação familiar. Relativamente à "satisfação com a família", os pais que têm bom suporte familiar apresentam menor bonding negativo. Esta relação pode ser comprovada pelo estudo de Felizardo et al (2010) que mostra 0 aumento do envolvimento paterno com maior satisfação e bem-estar das famílias (FELIZARDO et al, 2010). Também Figueiredo et al (2008) Cit. por Brandão (2009) corroboram estes resultados para o bonding negativo na medida em que afirmam que as relações familiares e com a companheira são essenciais na fase de transição para a parentalidade, e quando se desenvolvem de forma positiva contribuem para melhor harmonia pessoal, facilitando a transição para a parentalidade.

Relativamente ao bonding not clear, verificamos a existência de correlações negativas em todas as subescalas de suporte social, havendo as seguintes correlações significativas: "satisfação com os amigos" ( $p=0,047)$ e "satisfação com a família" ( $p=0,029)$. Assim, verificamos que à medida que aumentam a "satisfação com a família" e a "satisfação com os amigos", o bonding not clear diminui. Após a realização do modelo de regressão e aplicação dos testes $\mathrm{F}$ e t verificamos que os resultados não foram estatisticamente significativos, logo deduz-se que o suporte social não interfere com o bonding not clear. Realçamos que nenhuma subescala do suporte social é preditiva do bonding not clear.

Para corroborar os resultados obtidos no que diz respeito à correlação negativa entre a "satisfação com os amigos" e a "satisfação com a família" com o bonding not clear, referimos que o nascimento de um filho é uma experiência que envolve não só o casal mas também a família mais alargada. Este momento pode ser vivenciado de forma diferente para os pais e para as mães, dependendo da adaptação à nova situação, o que pressupõe as singularidades de cada um, as características individuais do bebé e as características do contexto social (MOURA-RAMOS; CANAVARRO, 2007, Cit. por BRANDÃO, 2009). Assim, infere Brandão (2009), a parentalidade desenvolve-se num contexto familiar, não descurando o enquadramento social e cultural de cada casal. Ray e Miller referem que a família apesar de apoiar emocionalmente, também pode constituir uma fonte de stress se 0 apoio prestado não for adequado. 0 apoio de amigos, como já foi referido, reveste-se de extrema importância pois estes constituem um suporte efetivo e essencial para 0 decurso da paternidade. Em suma, a importância do papel da família e amigos no apoio ao pai, minimiza o sentimento de ambivalência e incerteza, não interferindo no bonding not clear.

No que diz respeito ao bonding total, averiguamos a existência de correlações positivas em todas as subescalas do suporte social, e estatisticamente significativas a "satisfação com os amigos" ( $p=0,002)$, "intimidades" ( $p=0,004)$, "satisfação com a família" ( $p=0,000)$ e nas "atividades sociais" ( $p=0,003)$. À medida que aumentam os índices de suporte social, o bonding total aumenta. 


\section{A INFLUÊNCIA DO SUPORTE SOCIAL NA VINCULAÇÃO PAI/BEBÉ}

Aplicando o modelo de regressão, obtivemos que a primeira e única variável a entrar neste modelo foi a "satisfação com a família", justificando 17,8 \% do bonding total. Com a realização dos testes $T$ e f, verificamos que os resultados são estatisticamente significativos $(p=0,001)$ e pelos coeficientes padronizados, confirmamos que o maior valor preditivo está centrado na "satisfação com a família"; deduzindo assim que quanto maior a "satisfação com a família", maior é o bonding total. Estes dados são corroborados por Ribeiro (1999) Cit. por Moreira (2010) que refere, que a família é considerada como a maior fonte de suporte social. Neste sentido, Parke (1996) Cit. por Castoldi (2002), afirma que a relação do homem com a sua família de origem é um fator individual com impacto no envolvimento paterno. No estudo de Dessen e Braz (2000), acerca da rede social de apoio durante as transições familiares decorrentes do nascimento dos filhos, os autores verificam que 0 apoio psicológico recebido pela família, a aprovação dos parentes, as conversas e ajuda financeira fornecida pelos familiares são apontadas pelos pais como fatores positivos nas mudanças que ocorreram. Desta forma se infere, tendo em consideração Brandão (2009), que a experiência de ser pai desenvolve-se num contexto familiar, emergindo assim a importância de atender ao enquadramento social e cultural de cada casal.

Dos dados obtidos e de toda a bibliografia consultada podemos afirmar que o suporte social que o pai perceciona está intimamente relacionado com 0 envolvimento emocional que ele vai desenvolver com o seu bebé. Este processo tem início desde a conceção até ao nascimento, momento em que os profissionais de saúde assumem extrema importância na educação e estimulação do bonding paterno.

De facto, a gravidez, o parto e todo o processo inerente de transição para a parentalidade decorre num contexto sociocultural específico de cada pai e todos os fatores envolventes, como sejam a companheira, família, amigos, trabalho, atividades sociais vão interagir neste processo. Neste sentido, citamos Sroufe e Waters (1977) Cit. por Brandão (2009) que referem que o envolvimento emocional está diretamente relacionado com as respostas sociais, verbais e não verbais que reflectem a aceitação do recém-nascido pelo pai e vice-versa.

Para corroborar os dados empíricos referentes à influência do suporte social sob o bonding, mencionamos a opinião de Respler-Herman et al (2011) que afirma que os pais quando usufruem de maior apoio social estão melhor preparados para prestar cuidados e responder aos seus filhos de forma mais positiva. Ostberg e Hagekull (2000) Cit. por Respler-Herman (2011) afirmam ainda que os pais com elevado apoio social tendem a apresentar níveis menores de stress, o que conduz a uma parentalidade mais positiva e eficaz

\section{CONCLUSÕES}

A ideia de que o envolvimento emocional parental é uma circunstância determinante para a continuidade do estabelecimento do bonding entre esta díade, despertou-nos 0 interesse enquanto investigadores. Os resultados deste estudo são de extrema importância pois podem, com base numa reflexão crítica, produzir mudanças na nossa prática do cuidar o casal gravídico, promovendo um maior envolvimento emocional do pai.

Da investigação existente sobre este tema, constatamos que a vinculação paterna é um processo gradual de envolvimento afetivo do pai com o bebé, que se constrói com o desenvolvimento de determinadas competências. Foi nesta base que suportamos teoricamente o objetivo geral deste trabalho, o qual consistiu em estudar a relação entre o suporte social e o bonding na relação pai/bebé.

Esta investigação permitiu alcançar o objetivo a que nos propusemos, produzindo resultados relevantes para a prática do cuidar. Possibilitou ainda, identificar algumas relações estatisticamente significativas das diferentes variáveis com o bonding. 
Concluímos que quanto maior a satisfação com os amigos, maior é o bonding positivo e quanto maior é a satisfação com as intimidades, menor é o bonding negativo. Na relação entre bonding total e as subescalas do suporte social, inferimos que quanto maior a "satisfação com a família", maior é o bonding total. Os dados obtidos sugerem, na globalidade, que quanto maior for 0 apoio social percecionado pelo pai, maior será a sua predisposição para o estabelecimento de um bonding positivo.

Os enfermeiros devem olhar o pai tendo em consideração o contexto social em que está inserido, bem como o nível de suporte social que possui, disponibilizando-se para ajudar e esclarecer dúvidas já que os pais referem que o apoio dos enfermeiros, no ciclo gravídico e parto, é fulcral para que se desenvolva uma relação precoce e positiva entre a tríade pai/mãe/filho.

De facto os enfermeiros especialistas em saúde materna são privilegiados no que diz respeito ao acompanhamento do casal gravídico durante todo o processo de gravidez, trabalho de parto e parto, sendo importante que estes compreendam e interpretem os sentimentos expectantes do pai e planeiem os cuidados, envolvendo-o neste processo e atendendo às suas necessidades, sendo os resultados apurados relevantes para que possam basear as suas práticas nestas evidências científicas desenvolvendo competências que contribuam para a melhoria contínua dos cuidados.

\section{REFERÊNCIAS BIBLIOGRÁFICAS}

Bolli, A. C. B. (2002). 0 envolvimento paterno com o bebé e aos doze meses de idade (Dissertação de mestrado, Universidade do Rio Grande do Sul). Acedido em https://www.lume.ufrgs.br/bitstream/handle/10183/2553/000372062.pdf?sequence=1

Brandão, S. M. P. A. (2009). Envolvimento emocional do pai com o bebé: Impacto da experiência do parto (Dissertação de Mestrado, Universidade do Porto). Acedido em https://repositorio-aberto.up.pt/handle/10216/16151

Castoldi, L. (2002). A construção da paternidade desde a gestação até ao primeiro ano do bebé (Dissertação de mestrado, Universidade do Rio Grande do Sul). Acedido em https://www.lume.ufrgs.br/bitstream/handle/10183/1576/000351993.pdf?sequence $=1$

Deave, T., Johnson, D., \& Ingram, J. (2008). Transition to parenthood: The needs of parents in pregnancy and early parenthood. Pregnancy and Childbirth, 8, 30.

Dessen, M. A., \& Braz, M. P. (2000). Rede social de apoio durante transições familiares decorrentes do nascimento de filhos. Psicologia: Teoria e Pesquisa, (16)3, 221-231.

Felizardo, S. M. A. S. (2010). Os efeitos do suporte social em famílias de crianças com deficiência. In C. Nogueira, I. Silva, L. Lima, A.T. Almeida, R. Cabecinhas, R. Gomes, ... M.C. Taveira (Eds), Actas do VII Simpósio Nacional de Investigação em Psicologia (pp. 1-11). Viseu: Escola Superior de Educação de Viseu, I. P. V. Acedido em http://repositorio.ipv.pt/handle/10400.19/524

Figueiredo, B., Costa, R., \& Pacheco, A. (2005). Bonding: Escala para avaliar o envolvimento emocional dos pais com o bebé. Psychologica, 40, 133-154.

Genesoni, L., \& Tallandini, M. A. (2009). Men's psychological transition to datherhood: An analysis of the literature 1989-2008. Birth, (36)4, 305-318.

Menino, E., \& Sousa, C. (2010). E o pai?! As vivências do pai durante a primeira semana pós-parto. In Vulnerabilidades na gravidez e no pós-parto: Contributos (pp. 152-167).

Moreira, M. S. (2010) Stress e suporte social em pais de crianças com perturbações de hiperactiviade com défice de atenção (Dissertação de mestrado, Universidade Fernando Pessoa). Acedido em http://bdigital.ufp.pt/handle/10284/1418

Moura-Ramos, M., \& Canavarro, M. C. (2007). Adaptação parental ao nascimento de um filho: com- 


\section{A INFLUÊNCIA DO SUPORTE SOCIAL NA VINCULAÇÃO PAI/BEBÉ}

paração da reactividade emocional e psicossintomatologia entre pais e mães nos primeiros dias após o parto e oito meses após o parto. Análise Psicológica, (25)3, 399-413. Acedido em http://repositorio.ispa.pt/handle/10400.12/109

Oliveira, M. R. (2007). Nascimento de filhos: rede social de apoio e envolvimento de pais e avós (Dissertação de Mestrado, Universidade de Brasília). Acedido em http://repositorio.unb.br/handle/10482/2827?mode=full

Respler-Herman, M., Mowder, B. A., Yasik, A. E., \& Shamah, R. (2011). Parenting beliefs, parental stress, and social support relationship. Journal of Child and Family Studies, (21)2, 190-198. doi: 10.1007/s10826-011-9462-3

Ribeiro, J. L. P. (1999). Escala de satisfação com o suporte social. Análise Psicológica, 17(3), 547558. 